\title{
Editorial
}

\section{Automation, its Position in Industry, Social Areas and Education}

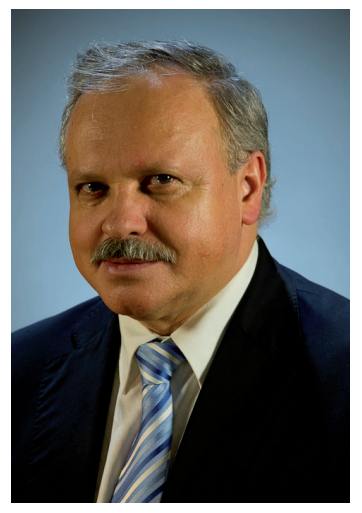

\author{
prof. Ing. Dušan Šimšík, PhD.
}

Technical University of Košice, Faculty of Mechanical Engineering, Department of Automation, Control and Human Machine Interactions

Prof. Ing. Dušan Šimšik, PhD. is full professor of automation and head of the Department of Automation, Control and Human Machine Interactions. He has published 6 monographs, 12 university text books and notebooks, and more than 400 publications in journals and conference proceedings in Slovakia and abroad $(218+187)$. His laboratories are devoted to industrial automation currently concentrating on wireless technology, but also on automation applied in human oriented sciences like in the forensic discipline - identification of persons using gait analysis, or in the application of ICT into public services monitoring and supporting systems for seniors and vulnerable persons based on domotic automation, monitoring of their daily activities, identification and prediction of critical situations mapping changes in their behaviour.

Automation is an interdisciplinary phenomena going through all areas of our life. The importance of automation has rapidly changed since Ford first significant jump into automated production. It is not only about research, because everything starts with a kind of research, not only about industry, but it is now about the implementation of automatic tools and procedures into public and private life too. Many automatic devices, even intelligent robots, ICT services are already on our market offering different levels of supporting automatic processes in our households, in communications with municipalities or governmental institutions.

Our Faculty of Mechanical Engineering is deeply involved in the newest movements in Slovak industry changes targeted on the automation nowadays often covered by the ideas of the Industry 4.0 program. The most important issue in that program plays connectivity of devices and equipment, as well as increasing automation. It leads to an increasing need for sensors to be able to monitor the state of the physical world, to understand its needs and gives a unique opportunity for traditional equipment and appliance manufacturers to transform their products into "smart things".

I will focus further on specific research areas that have been worked out in the recent period at our Department. Our Department of Automation, Control and Human Machine Interactions is also fully engaged in research program oriented on those progressive changes in industry for many years. Our experience is based on the international research cooperation in several EC financed projects in 6FP and 7FP programs devoted to the implementation of ICT into sophisticated automated devices like SMILING rehabilitation mechatronic shoes for gait training of seniors or ICT services supporting seniors and vulnerable persons living alone in 

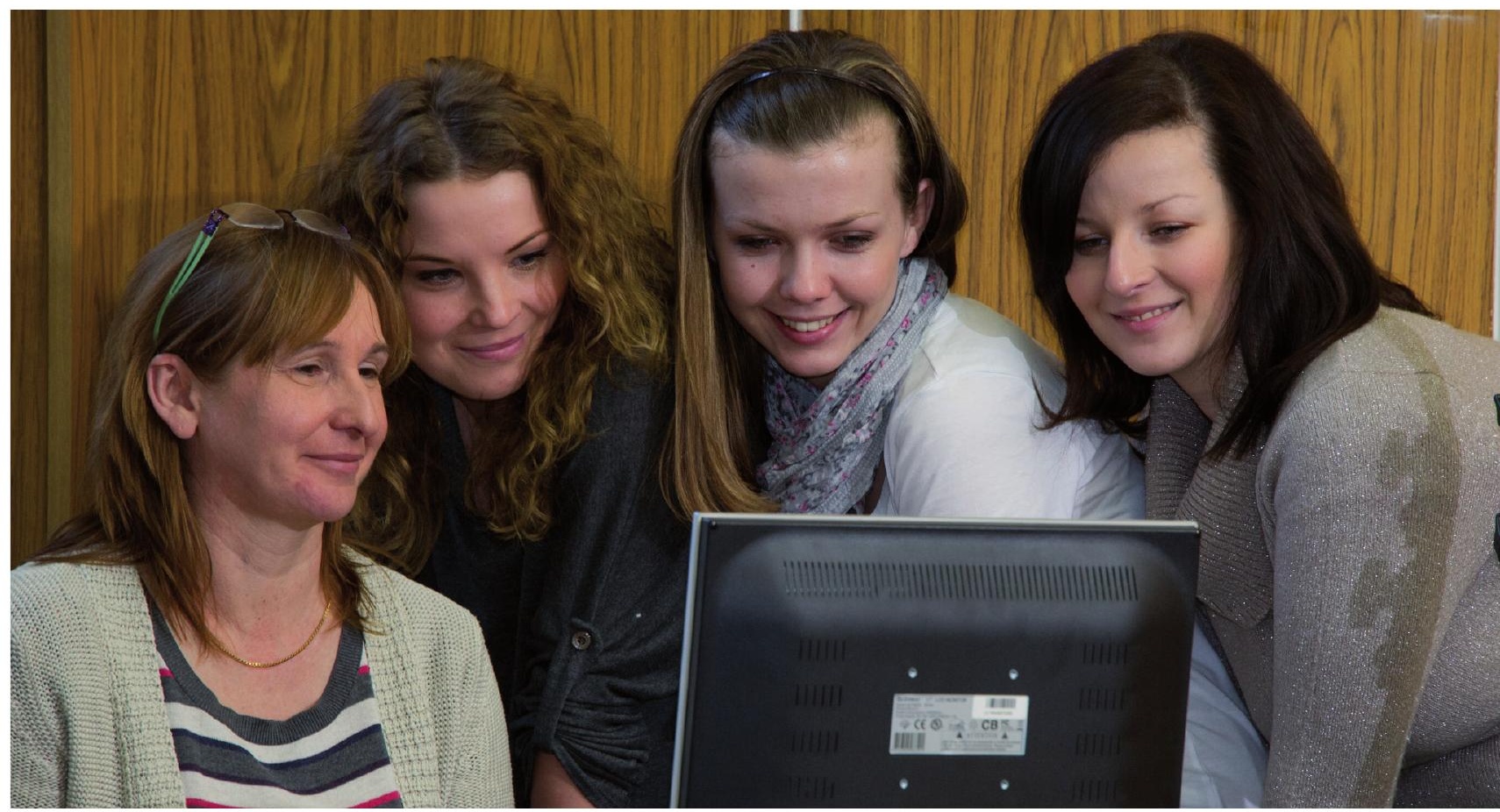

their homes in their daily activities enforcing them with higher level of social inclusion (internet, IP phones), safety and comfort, and other features leading to reach active and healthy ageing.

We work on experimental set up of sensors networks using wireless technology combined with cable communication preferably using 10 Link and Profinet protocols. Wireless technology will play important role in systems connecting a big amount of different devices, sensors, actuators by loT - Internet of things tools. It will include wearable devices in so called Body Area Networks, which have to be able now to communicate through IoT and offering Big Data for improving management of many processes in our working and private life. Therefore to implement IoT in our laboratories is a big challenge together with cloud technology. The loT enables physical objects to see, hear, think and perform jobs by having them communicate together, to share information and to coordinate decisions. Such technology is an important part of the Industry 4.0.

Cisco assumes year 50 milliards interconnected devices, Morgan Stanley 75 milliards until 2020. Big amount of sensors will generate Big data. IDC company anticipated 10 times more data in 2020 than in 2013 reaching the value 40 zettabytes (1021). When we will be able to evaluate such big data it will bring innovations, higher efficiency and productivity.

What are the most important challenges for experts in Automation? It is currently discussed at many forums in the European Union. Our Slovak Presidency prepared several international events to support research and its outputs implementation of automation, ICT and digitalisation into praxis to enhance the development of the European economy. For example, there are conferences Transition to Green Economy, ICT Proposers' Day, Bioeconomy Conference, Re-Industrialisation of the EU 2016, Social Sciences and Humanities: a New Agenda for Europe's Challenges, Central European Energy Conference with Energy Networking4Innovation. During all those events many matchmaking events will be organized to support Slovak experts entering into the international research platforms and projects.

Automation at our Mechanical Engineering Faculty is strongly supported by the management. Recently three separated Departments joined their human and technological potential in the Institute of Automation, Mechatronics and Robotics concentrated in one building. This created an integrated basis for more efficient laboratory work of our researchers, academic staff and students from three branches and study programs that will certainly facilitate our common research programs. 